\title{
CASO CLINICO: ASPERGILOSIS PULMONAR INVASIVA TRATADA CON VORICONAZOL EN PACIENTE CON SINDROME DE INMUNODEFICIENCIAADQUIRIDA
}

\author{
(Clinical case: invasive pulmonary aspergillosis treated with Voriconazol \\ in an AIDS patient)
}

\author{
H. Arias(1), R. Cruz (2), M.de la Prida (3), \\ W. Jensen (4), R. Ahumada (4), M. Huilcaman (4) \\ (1) Medicina Interna, Hospital Gustavo Fricke, (2) Cátedra de Micología \\ U de Valparaíso, (3) Respiratorio, Hospital Gustavo Fricke \\ (4) Infectología ,Hospital Gustavo Fricke \\ rcruzchoappa@gmail.com
}

Palabras clave: Aspergilosis invasiva, VIH, Voriconazol Key words: Invasive aspergillosis, VIH, Voriconazol

\section{RESUMEN}

Se reporta un caso clínico de aspergilosis pulmonar invasiva en un paciente de 29 años $\mathrm{VIH}(+)$ en etapa SIDA, sin antecedentes mórbidos conocidos, con diagnóstico inicial de neumonía por Pneumocystis jirovecii. Fue tratado con exito, pero sin asistir a controles posterior a su alta. Tres meses después ingresa al servicio de Urgencias del Hospital Gustavo Fricke con tos productiva mucopurulenta, disnea progresiva, fiebre intermitente y compromiso del estado general. La radiografía de tórax sugirió neumonía atípica, detectándose en los exámenes Pneumocystis jirovecii y Enterobacter aerógenes, por lo que se inicia tratamiento con Cotrimoxazol y Ertapenem. En los cultivos en agar Sabouraud se detectó abundante desarrollo de Aspergillus fumigatus, por lo que se empieza tratamiento con anfotericina $B$ en dosis crecientes hasta alcanzar $50 \mathrm{mg} /$ día, sin embargo, por reacciones adversas severas se decidió tratamiento con Voriconazol intravenoso y luego oral, con buena respuesta clínica, radiológica y de laboratorio. Es dado de alta con tratamiento con Voriconazol oral, además de profilaxis secundaria para P. jirovecii y Mycobaterium avium .

\section{INTRODUCCION}

Las infecciones fúngicas invasivas han aumentado en las últimas décadas debido al aumento de los pacientes inmunodeprimidos y a tratamientos médicos y quirúrgicos

Recibido el 21 Octubre 2008 Aceptado el 15 Diciembre 2008

\section{ABSTRACT}

A clinical case of an invasive pulmonary aspergillosis in a 29 aged VIH (+) patient, at an AIDS stage, lacking any known morbid data, and bearing an initial diagnosis of pneumonia by Pneumocystis jirovecii is herein described. Was successfully treated even though he failed to attend subsequent health controls. Three months later he is admitted in the Hospital Gustavo Fricke, showing productive mucupurulent cough, progressive disnea, intermittent fever and his overall health condition resulting deeply compromised. Thorax $X$-ray revealed an atypical pneumonia together with the presence of $\boldsymbol{P}$. jirovecii and Enterobacter aerogenes, and decided to treat him with Cotrimoxazol and Ertapenem. Meanwhile in agar cultures a heavy development of Aspergillus fumigatus was detected, thus the patient was given Anfotericina $B$ in increasing doses up to reach $50 \mathrm{mg} /$ day; however due to some severe adverse reactions, the treatment with intravenous and later oral Voriconazol, which rendered satisfactory clinical, radiological and laboratory responses was ultimately preferred. The patient is discharged from the hospital and advised to continue with oral Voriconazol besides undergoing secondary profilaxis for P. jirovecii and Mycobaterium avium.

agresivos (Bennet, 2005). Aproximadamente unas 20 especies de Aspergillus se han relacionado con patologías humanas. De éstas, las más importantes en frecuencia son A. fumigatus (80\% del total), A. flavus, A. niger, $\boldsymbol{A}$. nidulans y A. terreus (Thompson et al., 1987; Walsh \& Dixon, 1989; Herbrecht et al., 2002). La mayoría de estas 
especies esporulan profusamente con una alta dispersión anemófila de sus conidios, los cuales por su reducido tamaño, pueden ser inhalados causando multiplicación e invasión pulmonar o de otros órganos, en dependencia de la respuesta inmune del huésped (Bennet, 2005; Sanchez, 2000). Las aspergilosis invasivas presentan una alta mortalidad, la cual varía desde un 50\% hasta un $90 \%$ dependiendo de la localización de la infección, patología de base del paciente y de la precocidad del diagnóstico e inicio de tratamiento (Pontón, 2003). En la actualidad se cuenta con exámenes como el test de galactomanano y la tomografía axial computada (TAC), cuyo signo más precoz en aspergilosis pulmonar invasiva es el signo del halo. Estas pruebas, además de cultivos, examen microscópico directo, biopsia y biología molecular (Guzmán, 2005), permiten un diagnóstico oportuno en pacientes con factores de riesgo para tales infecciones. El patrón de referencia que permite establecer de forma probada, probable o posible la existencia de una infección fúngica por un hongo filamentoso, corresponde a los criterios conjuntos de la EORTC (European Organization for Research and Treatment of Cancer) y el Mycoses Study Group del NIAID (National Institute of Allergy and Infectious Diseases) de EE.UU (Pontón, 2003; Ascioglu et al., 2002).

Los factores de riesgo, criterios microbiológicos y clínicos para aspergilosis invasiva son variados, entre los factores de riesgo se destacan: (a) Menos de 500 neutrófilos $/ \mathrm{mm}^{3}$ por más de 10 días, (b) fiebre persistente (más de 96 hrs) refractaria a antibióticos de amplio espectro en pacientes de alto riesgo, (c) t emperatura mayor de $38^{\circ} \mathrm{C}$ o menor de $36^{\circ} \mathrm{C}$, (d) neutropénia por mas de 10 días en los 60 días anteriores, (e) uso de inmunosupresores en los 30 últimos días, (f) infección fúngica invasiva (probada o probable) durante el episodio neutropénico previo, (g) coexistencia de SIDA sintomático, (h) signos y síntomas de enfermedad injerto contra huésped severa o enfermedad extensa crónica, (j) uso prolongado (más de 3 semanas) de corticoides en los 60 días previos.

Dentro de los criterios microbiológicos en aspergilosis pulmonar se debe considerar: (a) cultivo positivo para Aspergillus spp. a partir de esputo o lavado broncoalveolar, (b) cultivo positivo o citología o microscopia directa positiva para Aspergillus spp. de esputo o lavado broncoalveolar, (c) antígenos de Aspergillus spp. positivo en muestras de lavado broncoalveolar, (d) citología o microscopia directa positiva para Aspergillus spp. en muestras habitualmente estériles.

Dentro de los criterios clínicos de infección pulmonar por Aspergillus se deben considerar: (a) criterios mayores: cualquiera de los siguientes hallazgos de la tomografía axial computarizada: signo del halo, signo del crecente aéreo (o signo de la media luna) o cavitación sin área de consolidación (excluyendo Mycobacterium, Legionella o Nocardia spp.) y (b) criterios menores: síntomas (tos, dolor torácico, hemoptisis, disnea), roce pleural, nueva opacidad radiológica, derrame pleural.

\section{CASO CLINICO}

Paciente de 29 años, soltero, homosexual, sin antecedentes mórbidos conocidos.

El 15 de Enero del 2008 tras cuadro de tos seca, baja de peso y disnea progresiva, es derivado desde el Hospital de Quintero con diagnóstico presuntivo de neumonía por Pneumocystis jirovecii. En esa oportunidad se detecta una LDH de 1046 UI/l, elevación de PCR y un infiltrado radiológico intersticial de ambos campos pulmonares, por lo que se inició tratamiento con Ceftriaxona y Cotrimoxazol E.V, el mismo que se mantuvo por 14 días hasta el alta, continuando con tratamiento oral. Durante la hospitalización se realizó un test de Elisa para VIH el cual resultó positivo y un recuento de CD4 de 14 células. El examen del lavado broncoalveolar no detectó presencia de Pneumocystis y el cultivo de Koch fue negativo.

El paciente no asistió a controles en policlínico de infectología hasta el 15 de abril del 2008, fecha en la que es derivado por médico particular al servicio de Urgencias del Hospital Gustavo Fricke con historia de cuadro de tos productiva mucopurulenta, disnea progresiva, fiebre intermitente y compromiso del estado general asociado a radiografía de tórax que sugirió neumonía atípica sin que se pudiera descartar un proceso tuberculoso (Fig. 1).

Ingresó al Hospital Gustavo Fricke el día 17 de Abril de 2008. Dentro del examen físico destacó leucoplasia vellosa, sin adenopatías cervicales, axilares ni inguinales, murmullo pulmonar disminuido globalmente y crepitantes bilaterales pero con predominio izquierdo y condilomas acuminados en región anal.

De los exámenes de laboratorio destacó una elevación significativa de PCR, no se solicitó LDH a su ingreso, leucocitos de $4.600 \mathrm{x} \mathrm{mm}^{3}$, hematocrito de $29 \%$, sin falla respiratoria hipoxémica y baciloscopías negativas. El día 18 de Abril se observó en muestra de expectoración y mediante tinción de azul de toluidina presencia de moderada cantidad de Pneumocystis jirovecii por lo que se inicia tratamiento con Cotrimoxazol ev.

Se solicitó fibrobroncoscopía la cual mostró una arquitectura conservada del árbol bronquial y abundante secreción mucopurulenta. El cultivo del LBA mostró desarrollo de Enterobacter aerógenes con un recuento de colonias significativo por lo que se inició tratamiento según antibiograma con Ertapenem a dosis de $1 \mathrm{~g}$ al día. Al mismo tiempo, los 6 cultivos en agar Sabouraud a $37^{\circ}$ mostraron abundante desarrollo de Aspergillus fumigatus 


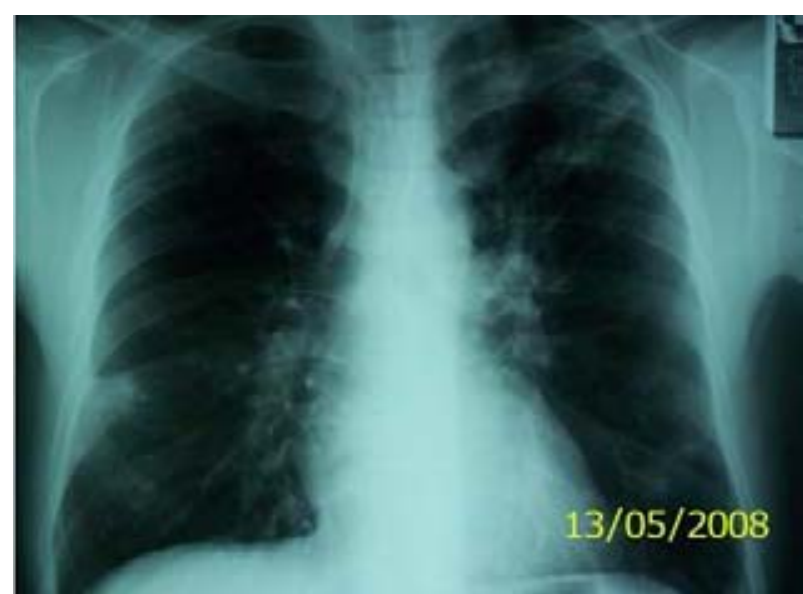

Fig. 1. Radiografía de tórax que sugirió neumonía

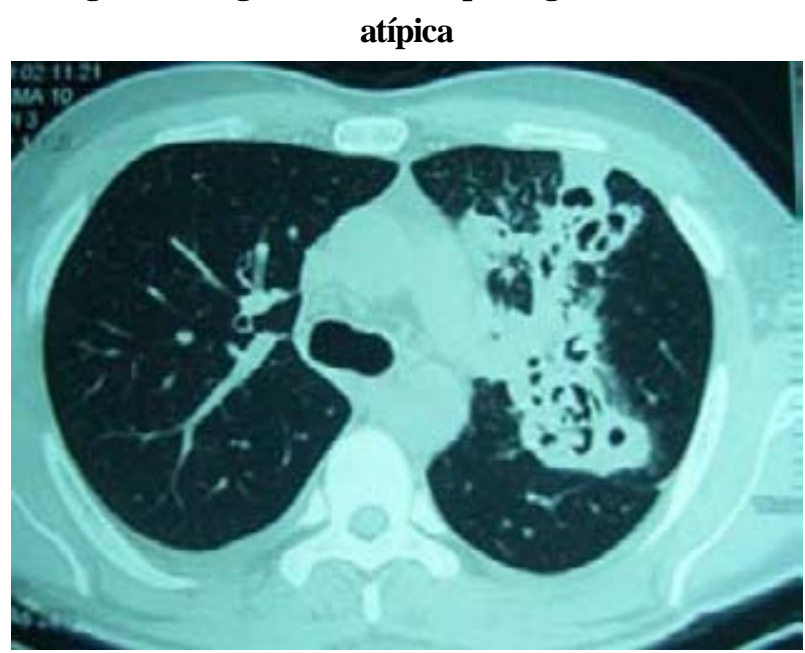

Fig. 3. TAC inicial con compromiso parenquimatoso bilateral, predominantemente en pulmon izquierdo

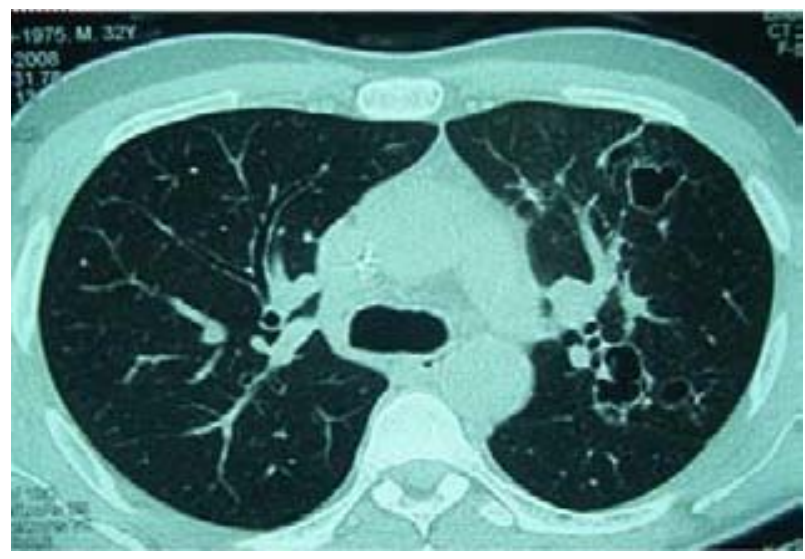

Fig. 5. TAC control de tratamiento

complex (Fig. 2), por lo que se inició tratamiento con Anfotericina B (AMB) en dosis crecientes hasta alcanzar los $50 \mathrm{mg} /$ día , tratamiento que se mantuvo por 10 días, tiempo en el cual presentó deterioro moderado de su

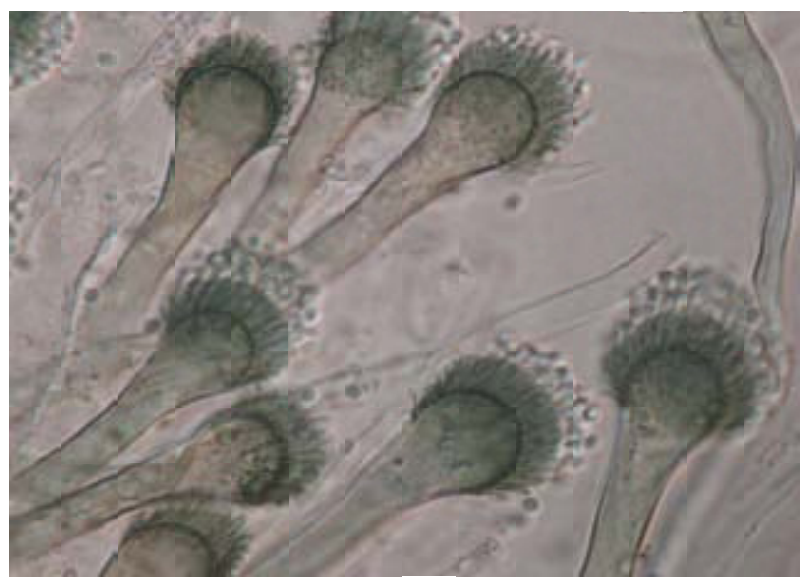

Fig. 2. Aspergillus Fumigatus

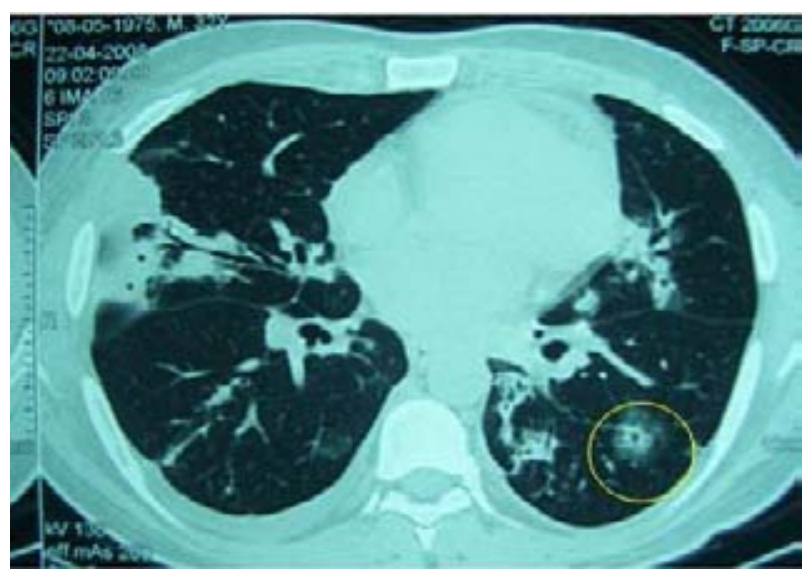

Fig 4. TAC de tórax que muestra signo del halo, característico de la aspergilosis pulmonar invasiva.

función renal, hipokalemia y datos que apoyaron una colestasia, por lo que se suspendió la Anfotericina y se inició terapia con Voriconazol en dosis de $6 \mathrm{mg} / \mathrm{kg}$ cada 12 horas el primer día y posteriormente $4 \mathrm{mg} / \mathrm{kg}$ cada 12 horas inicialmente por vía endovenosa.

El scanner de tórax inicial mostró lesiones pulmonares bilaterales, con compromiso predominantemente del parénquima pulmonar izquierdo (Fig.3) y lesión cavitada con signo del halo característico de aspergilosis pulmonar invasiva (Fig.4) y mínimo derrame pericárdico.

Finalmente el recuento de CD4 fue de 9 células por lo que se inició profilaxis para Mycobacterium avium con Azitromicina 500 mg semanal.

El paciente evolucionó favorablemente manteniendo parámetros sépticos apagados y clínicamente estable una vez iniciado el tratamiento. Se realiza TAC de tórax de control dos semanas posteriores a inicio de tratamiento, el cual muestró una resolución del patrón inflamatorio parenquimatoso y cavitaciones secuelares (neumatoceles) (Fig. 5). 
Es dado de alta con indicación de tratamiento vía oral con Voriconazol en dosis de 200 mg cada 12 horas, además de profilaxis secundaria para Pneumocytis jirovecii (Cotrimoxazol forte un comprimido diario) y Mycobaterium avium (Azitromicina $500 \mathrm{mg}$ semanal).

\section{Estudio micológico.}

Los cultivos de expectoración fueron enviados a la Cátedra de Micología de la Universidad de Valparaíso. En agar Sabouraud a $37^{\circ}$ crecieron colonias que tanto a la macro como a la microscopia eran sugerentes de Aspergillus fumigatus, las cuales se traspasaron a agar Czapec extracto de levadura (CYA) y agar Malta (MEA) a 25 y $37^{\circ} \mathrm{C}^{\circ}$. A $25^{\circ} \mathrm{C}$ en CYA durante 7 días, crecieron colonias entre 45 y $65 \mathrm{~mm}$ de diámetro, mientras en MEA a la misma temperatura 40-60 mm. En CYA a $37^{\circ} \mathrm{C}(60-66 \mathrm{~mm})$ mientras en MEA (62-64 mm). Máximo crecimiento $52^{\circ} \mathrm{C}\left( \pm 1^{\circ}\right)$. Conidios verde oscuros a verde turquesa, micelio blanco, con exudado y sin pigmentos, reverso amarillento en CYA. A la microscopía, las cabezas conidiales fueron predominantemente columnares, con conidióforo incoloro, de pared lisa, grises cerca del ápice, vesículas piriformes a espatuladas, entre 18-25 $\mu \mathrm{m}$; fiálides uniseriadas entre 5-9 por 2-3 $\mu \mathrm{m}$ cubriendo la mitad superior de las vesícula; conidios, globosos a elipsoidales, lisos a finamente rugosos 2-3 $\mu \mathrm{m}$.

Con estas características morfofisiológicas se confirmó el aislado como Aspergillus fumigatus

\section{DISCUSION}

Distintas especies del género Aspergillus pueden colonizar las vías respiratorias y posteriormente producir una invasión en aquellos pacientes con algún grado de inmunodepresión celular, en especial los neutropénicos. A. fumigatus es la especie más aislada en todas las formas clínicas de aspergilosis, tanto en la no invasiva como en la invasiva (Henderson \& Chapman, 2004; Cruz et al., 2005 ). Esta última destaca por su mayor gravedad y por su alta mortalidad dependiendo de la condición inmune del paciente, el diagnóstico precoz y el oportuno tratamiento con antifúngico de última generación. El cuadro clínico de una aspergilosis pulmonar es inespecífico y puede confundirse con una neumonía bacteriana o viral (Arteaga \& Grande Argudo, 1999). Las imágenes en la radiografía son variables y difíciles de diferenciar en su etiología, razón por la cual es urgente en estos pacientes, poder contar con TAC y test de galactomanano, el cual ha demostrado su mayor utilidad diagnóstica en pacientes neutropénicos que cursan precozmente una aspergilosis invasiva, además de su utilidad pronostica en el control de la respuesta al tratamiento antifúngico (Pereira et al., 2005; Pontón, 2003).

Con respecto al tratamiento, en los últimos años han aparecido nuevos medicamentos que han demostrado una mejor respuesta clínica y con menos reacciones adversas que la Anfotericina B deoxicolato, entre ellos los azoles de ultima generación como el Voriconazol, las Equinocandinas como la Caspofungina y las formulaciones lipídicas de Anfotericina B como la liposomal (Saballs et al., 2000; Wieland et al., 2005). El Voriconazol es considerado como la droga de elección en la aspergilosis invasiva; es un Triazol de segunda generación, derivado sintético del Fluconazol que actúa inhibiendo la 14-alfa demetilasa y la 24 dihidrolanosterol demetilasa, disminuyendo la síntesis de ergosterol, componente fundamental de la membrana celular fúngica(Wieland et al., 2005). La casuística nacional referente a aspergilosis pulmonar se inicia en la mitad del siglo pasado, evolucionando desde aportes del diagnóstico basados solo en hallazgos microbiológicos e histológicos (estos últimos principalmente post mortem) a los primeros intentos de anticuerpos anti Aspergillus en el suero de los enfermos (Yarzabal et al., 1974). Posteriormente se han publicado varios casos de aspergilosis principalmente invasivas (Thompson et al., 1985; Mendoza et al., 1985; Pillaux, et al., 2000; Zambrano et al., 2007 ), además de los aspectos anatomopatológicos, clínicos y técnicas modernas como PCR, que apoyan al diagnóstico convencional (Oddo \& Thompson, 1990).

El caso expuesto evidencia lo importante de la sospecha precoz de aspergilosis en los pacientes con SIDA, su diagnóstico oportuno con cultivos e imagenología, además de la buena respuesta al tratamiento con Voriconazol y las reacciones adversas severas de la Anfotericina B deoxicolato (falla renal, colestasia e hipokalemia).

Los métodos más empleados en los laboratorios en la identificación de las especies de Aspergillus de origen clínico, se basan mayoritariamente en el análisis de sus características de cultivo, propiedades fisiológicas y la morfología de sus estructuras asexuales o sexuales. Sin embargo, los esquemas morfológicos pueden presentar ciertas dificultadas cuando las cepas clínicas importantes y comunes, muestran grados de variabilidad en sus caracteres fenotípicos, situación que dificulta la determinación a nivel de especie (Samson et al., 2006).

Los miembros de la sección Fumigati en particular, son unos de los ejemplos actuales a considerar por poseer características morfológicas que se superponen al incluir distintas especies genéticas dentro de una única morfoespecie (Balajee et al.,2005a; 2005b; 2007; Katz et al., 2005). A. fumigatus sigue siendo el más común agente de aspergilosis, sin olvidar que sus aislamientos clinicos no son siempre morfológicamente característicos y se han cometido errores en el pasado en su identificación aplicando solo características morfológicas (Balajee et al., 2006). 
Estudios actuales, con métodos polifásicos (morfológicos, fisiológicos y moléculares), han reevaluado la sección Fumigati (así como otras) (Hong et al., 2005; Yaguchi et al., 2007) y sus resultados determinaron que las especies anamórficas de esta sección pueden ser divididas en 5 clades: la clade $\mathbf{I}$ incluye $\boldsymbol{A}$. fumigatus y sus otras 7 especies consideradas como sinónimos, la clade II: A.lentulus y A.fumisynnematus; la clade III: A. fumigatiaffinis y A. novofumigatus; la clade IV: especies atípicas de $A$. fumigatus incluyendo $A$. viridinutans y la clade $\mathrm{V}$ : A. brevipes, $\boldsymbol{A}$. duricaulis y $\boldsymbol{A}$. unilateralis. $\mathrm{La}$ mayoría de las cepas clínicas obtenidas en Japón se agrupan juntas en la clade I, otras en la clade II y IV, y ninguna en las clades III y V.

Se han analizado también las correlaciones entre morfología, máximo crecimiento a diversas temperaturas y la filogenia de los aislados dentro de la sección (Balajee et al. 2005a, 2007; Katz et al., 2005). El máximo desarrollo a diversas temperaturas de las clades I, II, III, IV y V fueron calculadas sobre los $50^{\circ} \mathrm{C}, 45^{\circ} \mathrm{C}, 45^{\circ} \mathrm{C}, 42^{\circ} \mathrm{C}$ y $42^{\circ} \mathrm{C}$, respectivamente; estos datos fisiológicos son muy útiles en la separación de especies dentro de la sección Fumigati, mostrando buena correlación entre las características filogenéticas y fenotípìcas (Balajee et al., 2007; Samson et al., 2007).

Nuestra cepa clínica, se clasificó morfofisiológicamente como $\boldsymbol{A}$. fumigatus mediante cultivos en medios estandarizados, creciendo a una temperatura máxima de unos $52^{\circ} \mathrm{C}$. A $25^{\circ} \mathrm{C}$ en MEA, presentó una morfología característica de sus vesículas, fiálides y conidios, por lo cual se separó de una especie similar capaz de crecer a la misma temperatura máxima, pero con una vesícula diferente como se observa en la nueva especie Aspergillus turcosus Hong, Frisvad \& Samson, aislada en Korea del Sur (en Samson et al., 2007).

\section{REFERENCIAS.}

Arteaga, E \& Grande Argudo, E. (1999). Aspergilosis pulmonar invasora en el síndrome de inmunodeficiencia adquirida. Rev. Iberoam. Micol. 16:211-215

Ascioglu, S.; Rex, J. H.; De Paw, B.; Bennett, J. E.; Bille, J.; Crokaert, F.; Denning, D.W. et al. (2002). Defining opportunistic invasive fungal infections in immunocompromised patients with cancer and hematopoietic stem cell transplants: an international consensus.. Clin. Infect. Dis. 34:7-14

Balajee, S.A; Gribskov, J.L.; Hanley, E.; Nickle, D.; Marr, K.A. (2005a). Aspergillus lentulus sp nov., a new sibling species of A. fumigatus. Eukaryotic Cell 4: 625-632

Balajee, S.A.; Gribskov, J.; Brandt, M.; Ito, J.; Fothergill, A.; Marr, K.A. (2005b). Mistaken identity: Neosartorya pseudofischeri and its anamorph masquerading as Aspergillus fumigatus. Journal of Clinical Microbiology 43:5996-5999
Balajee, S.A.; Nickle, D.; Varga, J.; Marr, K.A. (2006) Molecular studies reveal frequent misidentification of Aspergillus fumigatus by morphotyping. Eukaryotic Cell 5:1705-1712

Balajee, S.A.; Houbraken, J.;. Verweij, P.E.; Hong, S-B.; Yaghuchi, T.; Varga, J.; Samson, R.A. (2007). Aspergillus species identification in the clinical setting. Studies in Mycology 59:39-46

Bennet, J. (2005). Harrison, Principios de Medicina Interna Mcgraw-Hill $16^{\text {a }}$ edición.

Cruz, R.; Barthel, E.; Piontelli, E. \& Fernandez, G. (2005). Reportes Clínicos: infección rinosinusal probada por Aspergillus flavus y probable infección pulmonar por Emericella nidulans en pacientes inmunodeprimidos. Boletín Micológico. 20:109-115

Guzmán, D.A.M. (2005), Importancia del laboratorio en el diagnóstico de las micosis invasoras. Rev. Chil. Infect.21:39-47

Herbrecht, R.; Denning, D.W.; Patterson, T.F.; Bennett, J.E.; Greene, R.E.; Ostermann, J,M. (2002). Voriconazol versus anphoptericin B for primary of invasive aspergillosis. New Eng. J. Med. 347:408-414

Hong, S.B.; Go, S.J.; Shin, H.D.; Frisvad, J.C.; Samson, R.A. (2005). Polyphasic taxonomy of Aspergillus fumigatus and related species. Mycologia 97:1316-1329

Katz, M.E.; Dougall, A.M.; Weeks, K.\& Cheetham, B.F. (2005). Multiple genetically distinct groups revealed among clinical isolates identified as atypical Aspergillus fumigatus. Journal of Clinical Microbiology 43:551-555

Mendoza, F.; Retamal, C.; Ferrada, L.; Alvarez De Oro; Pivet, H.; Salamanca, L. (1985). Aspergilosis humana en Chile: estudio del último decenio. Farmacias. 17:8-11

Oddo, D. \& Thompson, L.(1990). Micosis pulmonares. Algunos aspectos de su diagnostico anatomopatologico y de laboratorio. Revista Chilena de Infectología. 7:197-207

Pontón, J. (Ed.) (2003). Aspergilosis Invasora, guía de bolsillo. Revista Iberoamericana de micología. http://www.reviberoammicol.com

Pereira, C; Nero, G; Lacaz, C.S; Machado, C.M. (2005). The contribution of galactomannan detection in the diagnosis of invasive aspergilosis in bone marrow transplant recipients. Mycopathology. 159:487-493

Pilleux, L.; Ponticas, M.; Carrasco,C.; León,A.; Salas,P.; Rodríguez,C.;Caorsi,I. (2000). Hialohifomicosis invasiva hepatoesplénica: Comunicación de un caso. Revista médica de Chile. vol.128. 6:641-646.

Reese, S.W. \& Betts. (2004) Enfermedades infecciosas. Editorial Marban

Samson, R.A.; Hong, S-B. \& Frisvald, C. (2006). Old and new concepts of species differentiation in Aspergillus. Medical Mycology 44: S133-S148

Samson, R.A.; Hong, S.; Peterson, S.W.; Frisvad, J.C.; Varga1, J. (2007). Poly-phasic taxonomy of Aspergillus section Fumigati and its teleomorph Neosartorya. Study in Mycology 


$$
\text { 59:147-203 }
$$

Wieland, T.; Liebold, A.; Jagiello, M.; Retal, G.; Birnbaum, D. (2005). Superiority of voriconazol over amfotericin B in treatment of invasive aspergillosis after heart transplantation. Journal of Heart and Lung Transplantation. 24: 102-104

Walsh, T.J.\&Dixon, D.N. (1989). Nosocomial aspergilosis: environmental microbiology, hospital epidemiology, diagnosis and treatment. Eur. J. Epidemiology 5:1616-1622

Yaguchi, T.; Horie, Y.; Tanaka, R.; Matsuzawa, T.; Ito, J.; Nishimura, K. (2007). Molecular phylogenetics of multiple genes on Aspergillus section Fumigati isolated from clinical specimens in Japan. Nippon Ishinkin Gakkai Zasshi 48:37-46

Yarzabal, L.; Sepúlveda,R.; Retamal, C.; Kinzel,R; Castro,M.; Salamanca, L. (1974). Aspergilosis respiratória humana. Rev. Médica Chile. 102:772-778
Saballs, P.; Lopez Colomes, J.L.; Gimeno Cobos, J.; Knobel, H. (2000). Tratamiento de la aspergilosis invasiva. Rev. Iberoam. Micol. 17S:93-96

Sanchez, J. (2000). Prevención de la aspergilosis nosocomial. Rev Iber de Micol.17S:100-102

Thompson, L.; Cinte, G.; Carvajao, L.C.; Oddo, D.; Paredes, P. (1985). Aspergilosis pulmonar invasiva em pacientes con leucemia linfoblástica aguda. Rev. Méd. Chile 113:345-352

Thompson, L.; Oddo, D. \& Retamal, C. (1987).Orientaciones clínicas y de laboratorio en micosis oportunistas. Rev. Chil. Infect. 4:69-74

Zambrano, A.; Biere, A.; Isamitt, D. (2007). Aspergilosis necrotizante crónica en un paciente con secuelas de tuberculosis pulmonar. Revista chilena de infectología. Enfermedades respiratorias. vol.23:43-48 